\title{
ПРОЕКЦИОННЫЙ МЕТОД СТОХАСТИЧЕСКОИ АППРОКСИМАЦИИ ДЛЯ ОТЫСКАНИЯ ДОПУСТИМОЙ ТОЧКИ В СТОХАСТИЧЕСКОМ ПРОГРАММИРОВАНИИ
}

1. Во многих методах решения экстремальных задач с ограничением предполагается, что известная начальная точка принадлежит допустимой области. В детерминированных задачах для нахождения точки $x$, удовлетворяющей неравенству $g(x) \leqslant 0$, разработано много методов (см., напр., $\left.\left[{ }^{1,2}\right]\right)$. В настоящей работе рассматривается один метод нахождения допустимого решения в задачах стохастического программирования. Требуется найти точку $x \in R^{r}$, удовлетворяющую неравенству

$$
\operatorname{Eg}(x, \xi) \leqslant 0,
$$

где $\xi-k$-мерный случайный вектор и $E-$ символ математического ожидания. Решение неравенства (1) основано на использовании отдельных реализаций случайного вектора когда функция $g(x, \xi)$ сепарабельна, т. е. когда

$$
g(x, \xi)=f(x)+h(\xi) .
$$

2. Для нахождения точки $x$ из множества

$$
Q=\{x: f(x)+E h(\xi) \leqslant 0\}
$$

предложим итерационный метод

$$
x_{n}=x_{n-1}-\gamma_{n}\left(x_{n-1}-P_{n}\left(x_{n-1}\right)\right), \quad n=1,2, \ldots,
$$

где $0 \leqslant \gamma_{n} \leqslant 1$, а $P_{n}(x)$ - проекция точки $x$ на множество $Q_{n}=$ $=\left\{x: f(x)+h\left(\xi_{n}\right) \leqslant 0\right\}$, т. е. $\left\|x-P_{n}(x)\right\|=\min _{y \in Q_{n}}\|x-y\|$ и $\xi_{n}-$ случайные величины, независимые и распределенные так же, как $\xi$.

Нахождение точки из множества $Q$ эквивалентно минимизации функции $I(x)=1 / 2\|x-P(x)\|^{2}$, где $P(x)$ - проекция точки $x$ на множество $Q$. Тогда $I(x) \geqslant 0$ и $Q=\{x: I(x)=0\}$. Приведем некоторые свойства операции проектирования и функции $I(x)$, которые нам понадобятся в дальнейшем. Предположим, что множество $Q$ выпукло и замкнуто.

Л ем м а 1. Вектор $x-P(x)$ является опорным $\kappa Q$ в точке $P(x)$, T. e.

$$
(x-P(x), y-P(x)) \leqslant 0, \quad y \in Q .
$$


Лемм а 2. Оператор проектирования является нерастягивающим, T. e.

$$
\|P(x)-P(y)\| \leqslant\|x-y\| .
$$

Доказательства лемм 1 и 2 см. в $\left[{ }^{2}\right]$.

Л емм а 3. Функция $I(x)$ выпукла, дифференцируема $u \operatorname{grad} I(x)=$ $=x-P(x)$. Доказательство см. в $\left[{ }^{3}\right]$ (лемма 16.2 , с. 135$)$.

Перейдем теперь к исследованию свойств метода (3). Обозначим через $F_{n-1}$ наименьшую $\sigma$-алгебру, порожденную случайными величинами $x_{0}, x_{1}, \ldots, x_{n-1}$. Для дальнейшего изложения предположим, что множества $Q_{n}$ почти наверное (п. н.) не пусты, т. е.

$$
P\left[Q_{n}=\varnothing, n=1,2, \ldots\right]=0,
$$

и что $E\left\{\left\|P_{n}(x)\right\| \mid F_{n-1}\right\}<\infty$ и $E\left\{\left\|P_{n}(x)\right\|^{2} \mid F_{n-1}\right\}<\infty$.

Л ем а 4. Если функция $f(x)$ выпукла и дифференцируема, то

$$
E\left\{P_{n}\left(x_{n-1}\right) \mid F_{n-1}\right\} \in Q .
$$

Доказательство. Прежде всего заметим, что в силу условий, наложенных на функцию $f(x)$, множества $Q_{n}$ выпуклы и замкнуты при всех $n=1,2, \ldots$. Следовательно, проекции $P_{n}(x)$ существуют и единственны для почти всех $x$ (см., напр., $\left[{ }^{4}\right]$, с. 286$)$, поэтому метод (3) имеет смысл. Из выпуклости и дифференцируемости функции $f(x)$ получим, что

$$
\begin{aligned}
& f\left(P_{n}\left(x_{n-1}\right)\right)+h\left(\xi_{n}\right) \geqslant f\left(E\left\{P_{n}\left(x_{n-1}\right) \mid F_{n-1}\right\}\right)+h\left(\xi_{n}\right)+ \\
+ & \left(f^{\prime}\left(E\left\{P_{n}\left(x_{n-1}\right) \mid F_{n-1}\right\}\right), P_{n}\left(x_{n-1}\right)-E\left\{P_{n}\left(x_{n-1}\right) \mid F_{n-1}\right\}\right) .
\end{aligned}
$$

Возьмем из этого неравенства условное математическое ожидание относительно $\sigma$-алгебры $F_{n-1}$ :

$$
\begin{aligned}
& E\left\{f\left(P_{n}\left(x_{n-1}\right) \mid F_{n-1}\right\}+E\left\{h\left(\xi_{n}\right) \mid F_{n-1}\right\} \geqslant E\left\{f\left(E\left\{P_{n}\left(x_{n-1}\right) \mid F_{n-1}\right\}\right) \mid F_{n-1}\right\}+\right. \\
+ & E\left\{h\left(\xi_{n}\right) \mid F_{n-1}\right\}+E\left\{\left(f^{\prime}\left(E\left\{P_{n}\left(x_{n-1}\right) \mid F_{n-1}\right\}\right),\right.\right. \\
& \left.\left.P_{n}\left(x_{n-1}\right)-E\left\{P_{n}\left(x_{n-1}\right) \mid F_{n-1}\right\}\right) \mid F_{n-1}\right\}=f\left(E\left\{P_{n}\left(x_{n-1}\right) \mid F_{n-1}\right\}\right)+E h(\xi)+ \\
+ & \left(f^{\prime}\left(E\left\{P_{n}\left(x_{n-1}\right) \mid F_{n-1}\right\}\right), E\left\{P_{n}\left(x_{n-1}\right) \mid F_{n-1}\right\}-E\left\{P_{n}\left(x_{n-1}\right) \mid F_{n-1}\right\}\right)= \\
= & f\left(E\left\{P_{n}\left(x_{n-1}\right) \mid F_{n-1}\right\}\right)+E h(\xi) .
\end{aligned}
$$

Так как

и

$$
f\left(P_{n}\left(x_{n-1}\right)\right)+h\left(\xi_{n}\right) \leqslant 0 \quad \text { при } \quad x_{n-1} \in Q_{n}
$$$$
f\left(P_{n}\left(x_{n-1}\right)\right)+h\left(\xi_{n}\right)=0 \quad \text { при } \quad x_{n-1} \notin Q_{n},
$$

To

$$
f\left(E\left\{P_{n}\left(x_{n-1}\right) \mid F_{n-1}\right\}\right)+E h(\xi) \leqslant 0 \text {, т. е. } E\left\{P_{n}\left(x_{n-1}\right) \mid F_{n-1}\right\} \in Q .
$$

Лемма доказана.

В работе [5] для минимизации детерминированного гладкого функционала $F(x)$ рассмотрен итерационный метод вида $x_{n}=x_{n-1}-\gamma_{n} s_{n}$, где $\gamma_{n} \geqslant 0$ и $s_{n}$ - некоторое случайное направление движения, зависящее от всех предыдущих значений $x$ и от $n$. В $\left[{ }^{5}\right] s_{n}$ называется псевдоградиентом $F(x)$ в точке $x_{n-1}$, если

$$
\left(F^{\prime}\left(x_{n-1}\right), E\left\{s_{n} \mid F_{n-1}\right\}\right) \geqslant 0,
$$

т. е. если вектор $s_{n}$ в среднем направлен под острым углом к градиенту. 
Покажем, что вектор $x_{n-1}-P_{n}\left(x_{n-1}\right)$ является псевдоградиентом функции $I(x)=1 / 2\|x-P(x)\|^{2}$ в точке $x_{n-1}$.

Л емм а 5. Если функция $f(x)$ выпукла и дифференцируема, то

$$
\left(I^{\prime}\left(x_{n-1}\right), E\left\{x_{n-1}-P_{n}\left(x_{n-1}\right) \mid F_{n-1}\right\}\right) \geqslant 0 .
$$
но,

Д ок а за те льст в о. По лемме $3 I^{\prime}(x)=x-P(x)$. Следователь-

$$
\begin{gathered}
\left(I^{\prime}\left(x_{n-1}\right), E\left\{x_{n-1}-P_{n}\left(x_{n-1}\right) \mid F_{n-1}\right\}\right)= \\
=\left(x_{n-1}-P\left(x_{n-1}\right), x_{n-1}-E\left\{P_{n}\left(x_{n-1}\right) \mid F_{n-1}\right\}\right)= \\
=\left\|x_{n-1}-P\left(x_{n-1}\right)\right\|^{2}+\left(x_{n-1}-P\left(x_{n-1}\right), \quad P\left(x_{n-1}\right)-E\left\{P_{n}\left(x_{n-1}\right) \mid F_{n-1}\right\}\right) \geqslant 0,
\end{gathered}
$$

так как по лемме $4 E\left\{P_{n}\left(x_{n-1}\right) \mid F_{n-1}\right\} \in Q$ и по лемме $1\left(x_{n-1}-P\left(x_{n-1}\right)\right.$, $\left.P\left(x_{n-1}\right)-E\left\{P_{n}\left(x_{n-1}\right) \mid F_{n-1}\right\}\right) \geqslant 0$. Лемма доказана.

Оценим теперь псевдоградиент $x_{n-1}-P_{n}\left(x_{n-1}\right)$.

Л е м а 6. Для псевдоградиента $x_{n-1}-P_{n}\left(x_{n-1}\right)$ справедлива оценка

$$
\left\|x_{n-1}-P_{n}\left(x_{n-1}\right)\right\| \leqslant\left\|x_{0}-P_{n}\left(x_{0}\right)\right\| .
$$

Д ок а з а те льст в о. Для любых $h\left(\xi_{n-1}\right)$ и $h\left(\xi_{n}\right)$ либо 1) $Q_{n} \subset Q_{n-1}$, либо 2) $Q_{n-1} \subset Q_{n}$ в силу сепарабельности функции $g(x, \xi)=$ $=f(x)+h(\xi)$. Если выполнено включение 1$)$, то

$$
\begin{aligned}
& \left\|x_{n-1}-P_{n}\left(x_{n-1}\right)\right\| \leqslant\left\|x_{n-1}-P_{n}\left(x_{n-2}\right)\right\|= \\
= & \left\|x_{n-2}-\gamma_{n-1}\left(x_{n-2}-P_{n-1}\left(x_{n-2}\right)\right)-P_{n}\left(x_{n-2}\right)\right\|= \\
= & \left\|\left(1-\gamma_{n-1}\right) x_{n-2}+\gamma_{n-1} P_{n-1}\left(x_{n-2}\right)-P_{n}\left(x_{n-2}\right)\right\|= \\
= & \left\|\left(1-\gamma_{n-1}\right)\left(x_{n-2}-P_{n}\left(x_{n-2}\right)\right)+\gamma_{n-1}\left(P_{n-1}\left(x_{n-2}\right)-P_{n}\left(x_{n-2}\right)\right)\right\| \leqslant \\
\leqslant & \left(1-\gamma_{n-1}\right)\left\|x_{n-2}-P_{n}\left(x_{n-2}\right)\right\|+\gamma_{n-1}\left\|P_{n-1}\left(x_{n-2}\right)-P_{n}\left(x_{n-2}\right)\right\|= \\
= & \left(1-\gamma_{n-1}\right)\left\|x_{n-2}-P_{n}\left(x_{n-2}\right)\right\|+\gamma_{n-1}\left\|P_{n-1}\left(x_{n-2}\right)-P_{n-1}\left(P_{n}\left(x_{n-2}\right)\right)\right\| \leqslant \\
\leqslant & \left(1-\gamma_{n-1}\right)\left\|x_{n-2}-P_{n}\left(x_{n-2}\right)\right\|+\gamma_{n-1}\left\|x_{n-2}-P_{n}\left(x_{n-2}\right)\right\|= \\
= & \left\|x_{n-2}-P_{n}\left(x_{n-2}\right)\right\| .
\end{aligned}
$$

Последнее неравенство имеет место в силу леммы 2. Если выполнено включение 2), то в силу выпуклости функции $\|x-P(x)\|^{2}$ имеем

$$
\begin{aligned}
& \left\|x_{n-1}-P_{n}\left(x_{n-1}\right)\right\|^{2} \leqslant\left\|x_{n-2}-P_{n}\left(x_{n-2}\right)\right\|^{2}- \\
- & 2 \gamma_{n-1}\left(x_{n-1}-P_{n}\left(x_{n-1}\right), x_{n-2}-P_{n-1}\left(x_{n-2}\right)\right)= \\
= & \left\|x_{n-2}-P_{n}\left(x_{n-2}\right)\right\|^{2}-2 \gamma_{n-1}\left(x_{n-1}-P_{n}\left(x_{n-1}\right), P_{n}\left(x_{n-1}\right)-P_{n-1}\left(x_{n-2}\right)\right)- \\
- & 2 \gamma_{n-1}\left(x_{n-1}-P_{n}\left(x_{n-1}\right), x_{n-2}-P_{n}\left(x_{n-1}\right)\right) \leqslant \\
\leqslant & \left\|x_{n-2}-P_{n}\left(x_{n-2}\right)\right\|^{2}-2 \gamma_{n-1}\left(x_{n-1}-P_{n}\left(x_{n-1}\right), x_{n-2}-P_{n}\left(x_{n-1}\right)\right) .
\end{aligned}
$$

Выполнение последнего неравенства гарантируется леммой 1. Покажем, что $\left(x_{n-1}-P_{n}\left(x_{n-1}\right), x_{n-2}-P_{n}\left(x_{n-1}\right)\right) \geqslant 0$. Можем записать

$$
\begin{gathered}
\left(x_{n-1}-P_{n-1}\left(x_{n-2}\right), x_{n-1}-P_{n}\left(x_{n-1}\right)\right)=\left\|x_{n-1}-P_{n}\left(x_{n-1}\right)\right\|^{2}+ \\
+\left(P_{n}\left(x_{n-1}\right)-P_{n-1}\left(x_{n-2}\right), x_{n-1}-P_{n}\left(x_{n-1}\right)\right) \geqslant 0,
\end{gathered}
$$

так как $P_{n-1}\left(x_{n-2}\right) \in Q_{n}$. Но 


$$
\begin{gathered}
x_{n-1}-P_{n-1}\left(x_{n-2}\right)=\left(1-\gamma_{n-1}\right) x_{n-2}+\gamma_{n-1} P_{n-1}\left(x_{n-2}\right)-P_{n-1}\left(x_{n-2}\right)= \\
=\left(1-\gamma_{n-1}\right)\left(x_{n-2}-P_{n-1}\left(x_{n-2}\right)\right)
\end{gathered}
$$

и

$$
\begin{aligned}
x_{n-2}-x_{n-1}= & x_{n-2}-\left(1-\gamma_{n-1}\right) x_{n-2}-\gamma_{n-1} P_{n-1}\left(x_{n-2}\right)= \\
& =\gamma_{n-1}\left(x_{n-2}-P_{n-1}\left(x_{n-2}\right)\right) .
\end{aligned}
$$

Следовательно,

$$
\begin{gathered}
\left(x_{n-1}-P_{n-1}\left(x_{n-2}\right), x_{n-1}-P_{n}\left(x_{n-1}\right)\right)= \\
=\left(1-\gamma_{n-1}\right)\left(x_{n-2}-P_{n-1}\left(x_{n-2}\right), x_{n-1}-P_{n}\left(x_{n-1}\right)\right)= \\
=\frac{\left(1-\gamma_{n-1}\right)}{\gamma_{n-1}}\left(x_{n-2}-x_{n-1}, x_{n-1}-P_{n}\left(x_{n-1}\right)\right) \geqslant 0
\end{gathered}
$$

и так как $0 \leqslant \gamma_{n-1} \leqslant 1$, то при $\gamma_{n-1} \neq 0$

$$
\left(x_{n-2}-x_{n-1}, x_{n-1}-P_{n}\left(x_{n-1}\right)\right) \geqslant 0 \text {. }
$$

Теперь имеем

$$
\begin{gathered}
\left(x_{n-1}-P_{n}\left(x_{n-1}\right), x_{n-2}-P_{n}\left(x_{n-1}\right)\right)=\left(x_{n-1}-P_{n}\left(x_{n-1}\right), x_{n-2}-x_{n-1}\right)+ \\
+\left\|x_{n-1}-P_{n}\left(x_{n-1}\right)\right\|^{2} \geqslant 0 .
\end{gathered}
$$

Следовательно,

$$
\left\|x_{n-1}-P_{n}\left(x_{n-1}\right)\right\| \leqslant\left\|x_{n-2}-P_{n}\left(x_{n-2}\right)\right\| .
$$

Заметим, что при $\gamma_{n}=0$ последнее неравенство выполняется автоматически. Завершая доказательство, отметим, что при любых $\xi_{n-2}$ и $\xi_{n}$ либо $Q_{n} \subset Q_{n-2}$, либо $Q_{n-2} \subset Q_{n}$. Далее ход рассуждений повторяется.

Исходя из лемм 4-6 докажем следующую теорему.

Т е о р ем а. Если функция $g(x, \xi)$ сепарабельна, функция $f(x)$ выпукла $и$ дифференцируема, $E\left\{\left\|P_{n}\left(x_{0}\right)\right\|^{2} \mid F_{n-1}\right\} \leqslant K\left(x_{0}\right)<\infty$, $\sum_{n=1}^{\infty} \gamma_{n}=\infty, \sum_{n=1}^{\infty} \gamma_{n}^{2}<\infty, \quad 0 \leqslant \gamma_{n} \leqslant 1$, то последовательность $\left\{x_{n}\right\}$, определенная соотношением (3), п. н. такова, ито для нее существует предел $I\left(x_{n}\right) u$

$$
\lim _{n \rightarrow \infty} \varrho\left(x_{n}, Q\right) \stackrel{\text { п.п. }}{=} 0 \text {. }
$$

Док аз а тельство. В силу метода (3) имеем

$$
\begin{aligned}
& 1 / 2\left\|x_{n}-P\left(x_{n}\right)\right\|^{2} \leqslant 1 / 2\left\|x_{n}-P\left(x_{n-1}\right)\right\|^{2}=1 / 2\left\|x_{n-1}-P\left(x_{n-1}\right)\right\|^{2}- \\
& -\gamma_{n}\left(x_{n-1}-P\left(x_{n-1}\right), x_{n-1}-P_{n}\left(x_{n-1}\right)\right)+\gamma_{n}^{2} / 2\left\|x_{n-1}-P_{n}\left(x_{n-1}\right)\right\|^{2}
\end{aligned}
$$

или

$$
\begin{gathered}
I\left(x_{n}\right) \leqslant I\left(x_{n-1}\right)-\gamma_{n}\left(x_{n-1}-P\left(x_{n-1}\right), x_{n-1}-P_{n}\left(x_{n-1}\right)\right)+ \\
+\gamma_{n}^{2} / 2\left\|x_{n-1}-P_{n}\left(x_{n-1}\right)\right\|^{2},
\end{gathered}
$$

поскольку $I(x)=1 / 2\|x-P(x)\|^{2}$.

Возьмем условное математическое ожидание от обеих частей последнего неравенства относительно $\sigma$-алгебры $F_{n-1}$ : 


$$
\begin{gathered}
E\left\{I\left(x_{n}\right) \mid F_{n-1}\right\} \leqslant I\left(x_{n-1}\right)-\gamma_{n}\left(x_{n-1}-P\left(x_{n-1}\right), x_{n-1}-E\left\{P_{n}\left(x_{n-1}\right) \mid F_{n-1}\right\}\right)+ \\
+\gamma_{n}^{2} / 2 E\left\{\left\|x_{n-1}-P_{n}\left(x_{n-1}\right)\right\|^{2} \mid F_{n-1}\right\} .
\end{gathered}
$$

Введем обозначение

$$
K=\left\|x_{0}\right\|^{2}+K\left(x_{0}\right) .
$$

Используя результаты лемм 5 и 6 и неравенство $\left\|x_{0}-P_{n}\left(x_{0}\right)\right\|^{2} \leqslant$ $\leqslant 2\left\|x_{0}\right\|^{2}+2\left\|P_{n}\left(x_{0}\right)\right\|^{2}$, получим $E\left\{I\left(x_{n}\right) \mid F_{n-1}\right\} \leqslant I\left(x_{n-1}\right)+\gamma_{n}^{2} K$. На основании теоремы 1 из работы $\left[{ }^{6}\right]$ можно утверждать, что п. н. существует $\lim I\left(x_{n}\right)$ и математические ожидания $E I\left(x_{n}\right)$ равномерно ограничены

$$
E I\left(x_{n}\right) \leqslant a .
$$

Перейдем теперь к математическим ожиданиям в неравенстве (5):

$$
\begin{gathered}
E I\left(x_{n}\right) \leqslant E I\left(x_{n-1}\right)-\gamma_{n} E\left(x_{n-1}-P\left(x_{n-1}\right), x_{n-1}-E\left\{P_{n}\left(x_{n-1}\right) \mid F_{n-1}\right\}\right)+ \\
+\gamma_{n}^{2} / 2 E E\left\{\left\|x_{n-1}-P_{n}\left(x_{n-1}\right)\right\|^{2} \mid F_{n-1}\right\} .
\end{gathered}
$$

Просуммируем (7) по $n$ от 1 до $\infty$. В силу (6) и условий на $\sum_{n=1}^{\infty} \gamma_{n}$ и $\sum_{n=1}^{\infty} \gamma_{n}^{2} \quad$ получим, что $\sum_{n=1}^{\infty} \gamma_{n}^{2} E E\left\{\left\|x_{n-1}-P_{n}\left(x_{n-1}\right)\right\|^{2} \mid F_{n-1}\right\}<\infty$. Следовательно,

$$
-\sum_{n=1}^{\infty} \gamma_{n} E\left(x_{n-1}-P\left(x_{n-1}\right), x_{n-1}-E\left\{P_{n}\left(x_{n-1}\right) \mid F_{n-1}\right\}\right)>-\infty
$$

или

$$
\sum_{n=1}^{\infty} \gamma_{n} E\left(x_{n-1}-P\left(x_{n-1}\right), x_{n-1}-E\left\{P_{n}\left(x_{n-1}\right) \mid F_{n-1}\right\}\right)<\infty
$$

Ho

$$
\sum_{n=1}^{\infty} \gamma_{n}=\infty \text { и }\left(x_{n-1}-P\left(x_{n-1}\right), x_{n-1}-E\left\{P_{n}\left(x_{n-1}\right) \mid F_{n-1}\right\}\right) \geqslant 0 \text {, }
$$

поэтому из (8) следует, что найдется подпоследовательность $n_{i}$, для которой

$$
\lim _{n \rightarrow \infty} E\left(x_{n_{t}-1}-P\left(x_{n_{t}-1}\right), x_{n_{t-1}}-E\left\{P_{n_{t}}\left(x_{n_{t}-1}\right) \mid F_{n_{t}-1}\right\}\right)=0 .
$$

Значит, существует подпоследовательность $n_{j}$ такая, что п. н.

$$
\lim _{n \rightarrow \infty}\left(x_{n_{j}-1}-P\left(x_{n_{j}-1}\right), x_{n_{j}-1}-E\left\{P_{n_{j}}\left(x_{n_{j}-1}\right) \mid F_{n_{j}-1}\right\}\right)=0 .
$$

Учитывая лемму 5, придем к выводу, что

$$
\lim _{n \rightarrow \infty}\left(x_{n-1}-P\left(x_{n-1}\right), x_{n-1}-E\left\{P_{n}\left(x_{n-1}\right) \mid F_{n-1}\right\}\right) \stackrel{\text { ․․․ }}{=} 0 .
$$

В силу леммы 5 имеем

$$
\begin{gathered}
\left(x_{n-1}-P\left(x_{n-1}\right), x_{n-1}-E\left\{P_{n}\left(x_{n-1}\right) \mid F_{n-1}\right\}\right)= \\
=\left\|x_{n-1}-P\left(x_{n-1}\right)\right\|^{2}+\left(x_{n-1}-P\left(x_{n-1}\right), P\left(x_{n-1}\right)-E\left\{P_{n}\left(x_{n-1}\right) \mid F_{n-1}\right\}\right) \geqslant
\end{gathered}
$$




$$
\geqslant\left\|x_{n-1}-P\left(x_{n-1}\right)\right\|^{2} \text {. }
$$

Следовательно, если для любого $\varepsilon>0$

$$
I\left(x_{n-1}\right)=1 / 2\left\|x_{n-1}-P\left(x_{n-1}\right)\right\|^{2} \geqslant \varepsilon,
$$

To

$$
\left(x_{n-1}-P\left(x_{n-1}\right), x_{n-1}-E\left\{P_{n}\left(x_{n-1}\right) \mid F_{n-1}\right\}\right) \geqslant 2 \varepsilon
$$

и так как последовательность $I\left(x_{n}\right)$ п. н. имеет предел, то

или

$$
\lim _{n \rightarrow \infty} I\left(x_{n}\right)=\lim _{n \rightarrow \infty} 1 / 2\left\|x_{n}-P\left(x_{n}\right)\right\|^{2} \stackrel{\text { ㅍ.н. }}{=} 0
$$

$$
\lim _{n \rightarrow \infty} \varrho\left(x_{n}, Q\right) \stackrel{\text { ㅍ.н. }}{=} 0 \text {. }
$$

Теорема доказана.

Следств и е. Пусть в дополнение к условиям теоремь $\lim f(x)=$ $=+\infty$ при $\|x\| \rightarrow \infty$. Тогда последовательность $\left\{x_{n}\right\}$, определенная соотношением (3), п. н. сходится $\kappa$ некоторой точке множества $Q$.

Доказ ательство. Так как $f\left(P\left(x_{n}\right)\right)=-E h(\xi)=C$ при каждом $x_{n}$, то существует $L$ такое, что $\left\|P\left(x_{n}\right)\right\| \leqslant L$ при каждом $x_{n}$. Повторяя рассуждения леммы 6 для $E h(\xi)$ и $h\left(\xi_{n}\right)$, получим, что

$$
\left\|x_{n}-P\left(x_{n}\right)\right\| \leqslant\left\|x_{n-1}-P\left(x_{n-1}\right)\right\| \leqslant\left\|x_{0}-P\left(x_{0}\right)\right\| \leqslant M\left(x_{0}\right)<\infty .
$$

\section{Следовательно,}

$$
\left\|x_{n}\right\| \leqslant\left\|P\left(x_{n}\right)\right\|+\left\|x_{n}-P\left(x_{n}\right)\right\| \leqslant\left\|P\left(x_{n}\right)\right\|+\left\|x_{0}-P\left(x_{0}\right)\right\| \leqslant L+M\left(x_{0}\right) .
$$

Из ограниченной последовательности $\left\{x_{n}\right\}$ можно выбрать сходящуюся подпоследовательность $\left\{x_{n_{k}}\right\}, x_{n_{k}} \rightarrow x^{*}$. Но согласно теореме п. н. $I\left(x_{n_{k}}\right) \rightarrow 0$, т. е. $I\left(x^{*}\right)=0$. А поскольку п. н. существует $\lim I\left(x_{n}\right)$, то и $\lim I\left(x_{n}\right) \stackrel{\text { п.н. }}{=} 0$. Следовательно, $x_{n} \stackrel{\text { п.н. }}{\rightarrow} x^{*} \in Q$. Следствие доказано.

Рассмотренный метод (3) эффективен лишь в том случае, когда операция проектирования проста. В качестве примера рассмотрим следующую задачу: найти точку из множества

$$
Q=\{x:(a, x) \leqslant(b, E \xi)\}, \quad a, b, x, \xi \in R^{r} .
$$

Тогда

$$
P_{n}\left(x_{n-1}\right)=\left\{\begin{array}{lll}
x_{n-1}, & \text { если } & \left(a, x_{n-1}\right) \leqslant\left(b, \xi_{n}\right), \\
x_{n-1}-\frac{\left(a, x_{n-1}\right)-\left(b, \xi_{n}\right)}{\|a\|^{2}} a, & \text { если } & \left(a, x_{n-1}\right)>\left(b, \xi_{n}\right),
\end{array}\right.
$$

и

$$
x_{n}=\left\{\begin{array}{lll}
x_{n-1}, & \text { если } & \left(a, x_{n-1}\right) \leqslant\left(b, \xi_{n}\right), \\
x_{n-1}-\gamma_{n} \cdot \frac{\left(a, x_{n-1}\right)-\left(b, \xi_{n}\right)}{\|a\|^{2}} a, & \text { если } & \left(a, x_{n-1}\right)>\left(b, \xi_{n}\right) .
\end{array}\right.
$$

Автор признателен Т. Тобиасу за внимание к работе и И. Петерсену за ценные замечания.

1. К а р м а нов В. Г., Математическое программирование, М., 1974.

2. Гурин Л. Г., Поляк Б. Т., Р ай Э. В., ЖВМ и МФ, 7, № 6, 1211 (1967). 
3. Итеративные методы в теории игр и программировании. Под ред. В. З. Беленского и В. А. Волконского, М., 1974.

4. Бу рб аки Н., Топологические векторные пространства, М., 1959.

5. Поляк Б. Т., Цы пкин Я. З., Автоматика и телемеханика, № 3, 45 (1973).

6. Гл а ды ше в Е. Г., Теория вероятности и ее применения, Х, № 2, 297 (1965).

Институт кибернетики Академии наук Эстонской ССР
Поступила в редакцию 30/VI 1976

\section{R. $L E P P$}

\section{STOHHASTILISE APROKSIMATSIOONI PROJEKTSIOONIMEETOD STOHHASTILISE PLANEERIMISULESANDE LUBATAVA LAHENDI LEIDMISEKS}

Mingi punkti leidmiseks hulgast $Q=\{x: f(x)+E h(\xi) \leqslant 0\}$ esitatakse juhusliku vektori $\xi$ realisatsioonidel $\xi_{n}$ põhinev projektsioonimeetod. Projekteeritakse hulgale $Q_{n}=$ $=\left\{x: f(x)+h\left(\xi_{n}\right) \leqslant 0\right\}$. Esitatud meetodil saadud jada koondub tõenäosusega 1 hulga $Q$ mingiks punktiks.

\section{R. LEPP}

\section{PROJECTION METHOD OF STOCHASTIC APPROXIMATION FOR SEEKING A FEASIBLE SOLUTION IN STOCHASTIC PROGRAMMING}

A projection method based on the realization $\xi_{n}$ of random vector $\xi$ is presented for seeking a point from the set $Q=\{x: f(x)+E h(\xi) \leqslant 0\}$. The projection is realized on a set $Q_{n}=\left\{x: f(x)+h\left(\xi_{n}\right) \leqslant 0\right\}$. The convergence conditions of the method with probability 1 are presented. 